\title{
Research, Science and Ophtalmology in Portugal
}

\author{
Investigação, ciência e oftalmologia em Portugal
}

$\mathbf{F}$ Irom the clinical point of view ophthalmology in Portugal reached levels of quality and excellence that rival with the best reference centers in the world, with very skilled and distinguished professionals. Regarding research and scientific development, although it has been a long way, it seems clear that the scientific production indicators are still below the existing potential and infrastructure in the country.

The primary goal of the ophthalmologist is the best treatment possible for the patient. However, scientific research is an essential factor to the progress of societies, and reflexively to the welfare of populations and treatment of patients. For this and other reasons, scientific research should not be separated from clinical practice, one must feed the other.

The best way to ensure that ophthalmologists have and use at each moment the best information available is to know formal medical education, and create a comprehensive and scientific culture. This is the culture that allows us to understandand integrate quickly to the clinical practice new knowledge and new discoveries are published in scientific journals. On the other hand, this same culture can not fail to give the ophthalmologist a culture of collaboration and openness to science.

If it is true that some trials are one of the most visible components of clinical research that is on going in Portugal, we can not help but notice that many of the most significant scientific developments are made at the border and at the intersection of different disciplines.

It is in these areas that the most relevant questions come up, and that is the most fertile field for the most creative responses that we find. Often, the answers to the complex questions we face call for knowledge ranging from biology to physics, and for means and resources involving technologies and diverse engineering solutions. The answer to some of our daily clinic questions would not be possible without the support of engineers and their technological solutions that allow us to measure indicators.

The ambition of an ophthalmologist who wants to be a part of the world and contemporary society will not be fulfilled if they do not want to know and learn more about diseases affectingsight. More over, this desire will not be fulfilled if, as far as possible, there is no process of creating new knowledge that may benefit the patients.

I agree with the idea that scientific societies can play a key role in this process, not only sharing information to their members and associates, but also being active players in the promotion of knowledge and research. Be it in advanced training activities or in the active disclosure and participation in research projects. I include in this formula of participation not only the participation in competitive research projects, but also actions of raising funds that can be directed to scientific activities. The role of a modern scientific society goes through an active participation in many of these activities. As an example, I can mention the challenge associated to the active and healthy aging of populations. It is about responding to a challenge in which certainly clinical research and in particular the view of the sciences have a leading role, well illustrated by the number and severity of ocular diseases associated to aging.

As a scientific society, the Portuguese Society of Ophthalmology is not indifferent to these challenges, and more than federating the Portuguese ophthalmologists it contributes to the internationalization and differentiation of these doctors, keeping them informed and, wherever possible, involved in the most important studies and findings in Europe and worldwide.

This is the information thatone way or another, sooner or later, may improve the sight of our patients.

Maria João Quadrado

President of the Portuguese Society of Ophthalmology 